\title{
Procesos organizacionales de la gestión logística en las empresas distribuidoras de productos lácteos
}

\section{Organizational processes of logistics management in dairy product distributor companies}

\begin{tabular}{|r|r|}
\hline Karla Castellanos & Max Olivares \\
\cline { 2 - 2 } $\begin{array}{r}\text { karlazcastellanosm@gmail.com } \\
\text { ORCID: 0000-0001-6293-8158 } \\
\text { Universidad de Carabobo, } \\
\text { Valencia - Venezuela }\end{array}$ & direccion@cidecuador.org \\
ORCID: 0000-0002-6281-7951 \\
Centro de Investigación y Desarrollo, \\
Guayaquil - Ecuador
\end{tabular}

Artículo recibido en septiembre 2020 / Arbitrado en octubre 2020 / Aceptado en diciembre 2020 / Publicado en septiembre 2021

RESUMEN

Palabras clave:

ABSTRACT

Keywords:
El objetivo fue analizar el proceso de organización logística en las empresas distribuidoras de productos lácteos. La investigación se consideró de tipo descriptiva utilizando para ello un diseño no experimental, de campo y transeccional. La población estuvo conformada por nueve personas que ocupan los cargos de gerentes en las empresas de productos lácteos ubicadas en el municipio Cabimas y que se encontraron activas al momento de la investigación. Para el tratamiento de la información se estructuró un cuestionario conformado por nueve ítems con cinco alternativas de respuestas, validado por el juicio de expertos y cuya confiabilidad fue calculada a través del coeficiente de Alfa Cronbach, obteniéndose 0,949. Para el análisis estadístico, se aplicó la estadística descriptiva, a través de las frecuencias y la media aritmética. Se concluye que las empresas manejan una alta utilización de la estructura organizacional, división del trabajo, jerarquización y opciones organizacionales como proceso de organización logística.

División del trabajo, estructura organizacional, jerarquización, opciones organizacionales, proceso de organización logística

The objective was to analyze the logistical organization process in the dairy product distribution companies. The research was considered descriptive, using a non-experimental, field and transectional design. The population consisted of nine people who occupy the positions of managers in the dairy products companies located in the Cabimas municipality and who were active at the time of the investigation. For the treatment of the information, a questionnaire was structured consisting of nine items with five alternative responses, validated by the judgment of experts and whose reliability was calculated using the Alpha Cronbach coefficient, obtaining 0.949. For the statistical analysis, descriptive statistics was applied, through the frequencies and the arithmetic mean. It is concluded that the companies manage a high use of the organizational structure, division of labor, hierarchization and organizational options as a logistics organization process.

Division of labor, organizational structure, hierarchy, organizational options, logistics organization process 


\section{INTRODUCCIÓN}

Desde el punto de vista empresarial, la logística surge debido a las exigencias de la diversidad de empresas existentes para maximizar su rentabilidad, adquirir ventajas competitivas que le permitan posicionarse en mercados específicos, mediante un adecuado manejo de los recursos financieros, económicos, técnicos y humanos, principalmente, manteniendo de manera eficiente y organizada la comunicación con los entes involucrados del proceso logístico, específicamente con el cliente, los proveedores y los operarios.

En este orden de ideas, Ballou (2004), describe la gestión logística mediante los procesos de planificación, organización y control. En esencia, la gestión logística, es una parte esencial de la administración de las operaciones, puesto que enfoca sistemáticamente todas las áreas de la organización con el propósito de cumplir con cada una las actividades inherentes a esta gestión, mediante el manejo eficiente de los inputs que incluyen adquisición de materias primas, de recursos humanos, almacenamiento, administración de los inventarios, transporte, distribución, para lograr unos outpus de calidad, en el momento preciso y al menor costo posible.

Dentro de este contexto, existen en Venezuela, organizaciones productoras de alimentos de consumo masivo, que ven la gestión logística como un proceso que le permite afrontar las dificultades en la gestión de sus operaciones, tal y como sustenta Ávila (2010). Más aun, en el estado Zulia, específicamente en la Costa Oriental del Lago en el municipio Cabimas, existen empresas distribuidoras de productos lácteos, las cuales tienen como propósito almacenar y distribuir el producto terminado, entre las que se ubican: Upaca, Andi Cabimas y lácteos Yaracuy.

En estas empresas distribuidoras de productos lácteos, las investigadoras, pudieron constatar mediante entrevistas previas realizadas al personal gerencial y supervisorio, que presentan debilidades en el uso de la gestión logística, en lo referente a los procesos o funciones administrativas necesarias para la consecución de los objetivos organizacionales. Principalmente, se observa deficiencia en el desarrollo de la gerencia, puesto que no se cumplen en su totalidad las directrices, cada trabajador cumple funciones independientes sin llevar alguna coordinación. Por ende, la falla producida en la plataforma central de cualquier organización como lo es el personal directivo origina problemas en la toma de decisiones en todos los procesos administrativos, de las empresas en estudio.

De esta problemática surge la inquietud e interés en realizar el estudio acerca del proceso de organización logística en las empresas distribuidoras de productos lácteos, a través de su estructura organizacional, división del trabajo, jerarquización y opciones organizacionales.

\section{Proceso de organización logística}

Para Castellanos (2009), la gestión logística consiste en gerenciar estratégicamente la adquisición, el movimiento, el almacenamiento de productos y el control de inventarios, así como todo el flujo de información asociado, a través de los cuales la organización y su canal de distribución se encauzan de modo tal que la rentabilidad presente y futura de la empresa es maximizada en térmicos de costos y efectividad.

Refiere el citado autor, que para lograr un buen funcionamiento de la administración logística se necesita de la implementación de 
ciertas estrategias comparativas en actividades asociadas a los procesos logísticos (servicio al cliente, gestión de inventario, transporte y procesamiento de pedidos), para lograr la satisfacción del cliente y la reducción de costos, que es uno de los factores por lo que las organizaciones se enfocan en la logística.

Así las cosas, para Carranza y Sabria (2005), el proceso de la logística es el proceso de planear e implantar, controlar de manera eficiente y económica el flujo y almacenamiento de materias primas, inventarios en proceso, productos terminados e información vinculada con ellos desde el punto de origen al punto de consumo con el propósito de adecuarse a los requerimientos del cliente.

Ahora bien, dentro del proceso de la logística, el proceso de organización se enfoca en primer lugar en el esfuerzo de la logística, así pues, colocar en la empresa a las personas responsables de las actividades logísticas, de manera que se favorezca la coordinación entre ellas. Dichas disposiciones en la empresa promueven la eficiencia, el suministro y en la distribución de bienes y servicios mediante el estímulo de la compensación de costos, que a menudo se encuentra al planear y operar el sistema de la logística (Ballou, 2004).

Cabe destacar, la importancia de la estructura organizacional, considerada como la espina dorsal, el esqueleto que sustenta $y$ articula todas sus partes integrantes. En este sentido, la estructura organizacional representa una naturaleza predominantemente estática, referente a la configuración de los órganos y equipos de la organización (Chiavenato, 2007). Entonces, la estructura organizacional queda definida como la forma de dividir, organizar y coordinar las actividades de la organización.
Dentro de esta perspectiva, se considera fundamental mencionar la división del trabajo, ya que el trabajo se ejecuta con más eficiencia cuando se especializan los empleados. En efecto, consiste en dividir y fragmentar las tareas organizacionales en actividades separadas. Sin embargo, en algunas organizaciones se está abandonando este principio, ya que con tanta especialización, los empleados se mantienen aislados y realizan una tarea sencilla, repetitiva y monótona que provoca fatiga psicológica y alienación, debido a esto, las organizaciones amplían los cargos para proporcionar mayores desafíos, y asignan tareas a equipos para que los empleados se turnen en las diversas tareas desempeñadas, es decir, trabajan mediante el enfoque de equipos (Chiavenato, 2007).

Desde el punto de vista de la logística, la fragmentación de las actividades se da a través de tres áreas fundamentales: finanzas, operaciones y marketing. Por consiguiente, la responsabilidad de la transportación debe colocarse en operaciones, el inventario dividirse entre las tres funciones y el procesamiento de pedidos colocarse bajo marketing o finanzas. Todo lo anterior refleja, que se necesita de una estructura organizativa que ayude a la coordinación de la toma de decisiones referentes a diversas actividades de la logística (Ballou, 2004).

En el mismo marco de ideas, Chiavenato (2007), menciona a la jerarquización dentro del proceso de organización, definido como la disposición de las funciones de una organización por orden de rango, grado o importancia. Así pues, los niveles jerárquicos son el conjunto de órganos agrupados de acuerdo con el grado de autoridad y responsabilidad 
que posean, independientemente de la función que realicen. En tal sentido, la jerarquización implica la definición de la estructura de la empresa por medio del establecimiento de centros de autoridad (comunicación) que se relacionen entre sí con precisión y que ésta fluya desde el más alto ejecutivo hasta el nivel más bajo.

Siguiendo el mismo contexto, se anexa el criterio de Gavilanes (2012), al considerar los niveles jerárquicos como la dependencia y relación que tienen las personas dentro de la empresa. Principalmente en toda organización se cuenta con tres niveles, en primer lugar, el primer nivel es el directivo, cuyas funciones son, legislar políticas, crear normas y procedimientos que deben seguir la organización. Así como también realizar reglamentos, decretar resoluciones que permitan el mejor desenvolvimiento administrativo y operacional de la empresa.

El nivel ejecutivo es el segundo al mando de la organización, es el responsable del manejo de la misma, su función consiste en hacer cumplir las políticas, normas, reglamentos, leyes $y$ procedimientos que disponga el nivel directivo, en coordinación con el nivel operativo para su ejecución. Por consiguiente, el encargado de este nivel, velara el cumplimiento de las leyes y reglamentos obligatorios y necesarios para el funcionamiento de la empresa. Gavilanes (2012).

Finalmente, el tercer nivel más importante de la empresa y el responsable directo de la ejecución de las actividades básicas es el nivel operativo, siendo el pilar de la producción y la comercialización. Tiene el segundo grado de autoridad y es responsable del cumplimiento de las actividades encomendadas a la unidad, bajo su mando puede delegar autoridad, mas no responsabilidad (Gavilanes, 2012).
En este orden de ideas se puede citar la opinión de Ballou (2004), acerca de las opciones organizacionales, estas pueden clasificarse como de tipo informal, semi-formal y formal. En la organización informal, existe un cruce de actividades y una amplia coordinación entre las actividades y cooperación entre los responsables. En relación a la organización semi-formal, esta se considera matricial, puesto que el gerente de logística es responsable por todo el sistema logístico, pero no tiene autoridad directa sobre las actividades que lo componen. Por último, la organización formal, es aquella que establece líneas claras de autoridad y responsabilidad para la logística.

Al respecto Sierra (2013), menciona que la organización formal debe diseñar áreas, sectores y departamentos en los que se concentrarán funciones y tareas. Cada uno de ellos se vincula con los restantes a través de canales de comunicación, conformando una estructura planificada con distintos niveles de jerarquía, autoridad y responsabilidad. Si bien toda organización requiere una organización formal, previamente planificada y diseñada, coexiste con ella otro tipo de organización denominada organización informal, la misma es no prevista ni requerida, surge como consecuencia de relaciones personales y de amistad entre sus integrantes, grado de interacción de los mismos, intereses comunes, existencia de líderes naturales, fallas de la comunicación, entre otros.

Según Chiavenato (2007), la organización formal comprende estructura organizacional, directrices, normas y reglamentos de la organización, rutinas y procedimientos, en fin, todos los aspectos que expresan cómo la organización pretende que sean las relaciones entre los órganos, cargos y ocupantes, con la finalidad de que sus objetivos sean alcanzados $y$ 
su equilibrio interno sea mantenido. Este tipo de organizaciones, pueden a su vez, tener uno o más de los siguientes tipos de organización:

Refiere el citado autor, que, en primer lugar, la organización formal puede ser de tipo lineal en donde existen líneas directas y únicas de autoridad y responsabilidad entre superior $y$ subordinados. De ahí su formato piramidal. Cada gerente recibe y transmite todo lo que pasa en su área de competencia, pues las líneas de comunicación son estrictamente establecidas. Es una forma de organización típica de pequeñas empresas o de etapas iníciales de las organizaciones.

También se encuentran en las organizaciones formales, las de tipo funcional es el tipo de estructura organizacional que aplica el principio funcional $o$ principio de la especialización de las funciones. Muchas organizaciones de la antigüedad utilizaban el principio funcional para la diferenciación de actividades o funciones. El principio funcional separa, distingue y especializa: Es el germen del staff (Chiavenato, 2007).

Por último, menciona la organización LíneaStaff que es el resultado de la combinación de los tipos de organización lineal y funcional, buscando incrementar las ventajas de esos dos tipos de organización y reducir sus desventajas. En la organización línea-staff, existen características del tipo lineal y del tipo funcional, reunidas para proporcionar un tipo organizacional más complejo y completo.

En la misma línea de Chiavenato, (2007), en la organización línea-staff coexisten órganos de línea denominados órganos de ejecución y de asesoría llamados órganos de apoyo y de consultoría, manteniendo relaciones entre sí. Los órganos de línea se caracterizan por la autoridad lineal y por el principio escalar, mientras los órganos de staff prestan asesoría y servicios especializados.

Agrega el citado autor, que, en el contexto de las organizaciones informales, este tipo de organizaciones consiste en medios no oficiales pero que influyen en la comunicación, la toma de decisiones y el control que son parte de la forma habitual de hacer las cosas en una organización.

\section{MÉTODO}

Según el nivel de profundidad con el cual se abordó el problema, la investigación se adapta a la descriptiva, utilizando para ello un diseño no experimental, de campo y transeccional. La población quedo representada por los gerentes generales y las gerentes de línea de las empresas de productos lácteos activas al momento de la investigación, entre las cuales se encuentran: UPACA sede Cabimas, lácteos Los andes (Andi-Cabimas) y lácteos Yaracuy.

Para la recolección de la información, se aplicó un cuestionario contentivo de 9 ítems, con un escalamiento tipo Likert de cinco alternativas de respuestas: totalmente de acuerdo (5); medianamente de acuerdo (4); ni de acuerdo ni en desacuerdo (3); medianamente en desacuerdo (2); totalmente en desacuerdo (1). El mismo fue validado por el juicio de cinco expertos y cuya confiabilidad fue calculada a través del coeficiente de Alfa Cronbach, cuyo valor fue 0,949.

Seguidamente el análisis estadístico, se realizó a través de las frecuencias y la media aritmética. Para tal fin, la investigadora diseñó un baremo de interpretación para la media aritmética, representado en el cuadro 1 , donde se puede identificar el nivel de respuesta según el rango de apreciación establecido para cada intervalo. 
Cuadro 1. Categoría de análisis para la interpretación del promedio

\begin{tabular}{ccc}
\hline DIMENSIÓN & RANGO & NIVEL DE RESPUESTA \\
\hline & $4.21-5.00$ & Muy alta utilización \\
Proceso de Organización & $3.41-4.20$ & Alta utilización \\
& $2.61-3.40$ & Moderada utilización \\
$1.81-2.60$ & Baja utilización \\
& $1.00-1.80$ & No hay utilización \\
\hline
\end{tabular}

Fuente: Elaboración propia (2019)

\section{RESULTADOS}

En la tabla 1 , se describen los resultados del indicador estructura organizacional obteniendo un promedio de 4,22, lo cual se ubica en la categoría de muy alta utilización, lo que evidencia un manejo adecuado del indicador, donde el $77,78 \%$ de los encuestados están totalmente de acuerdo y solo un $22,22 \%$ está totalmente en desacuerdo en que la estructura organizacional se encuentra claramente dividida, con el propósito de establecer algunas directrices para dividir, organizar y coordinar las actividades de la organización, según lo citado por Chiavenato 2007).
De igual forma el indicador muestra con un $55,56 \%$ que los encuestados están totalmente de acuerdo, un 22,22\% medianamente de acuerdo y solo un $22,22 \%$ no está de acuerdo ni en desacuerdo en manifestar que las tareas en la organización están siendo ejecutadas por personal calificado, ubicándose en una categoría de muy alta aplicación con un promedio de 4,33, lo que indica que la empresa coloca a las personas responsables en los cargos para promover la eficiencia, el suministro y la distribución de bienes y servicios que a menudo se encuentra al planear y operar el sistema de la logística, coincidiendo con la teoría expuesta por Ballou (2004). 
Tabla 1. Indicador: Estructura organizacional

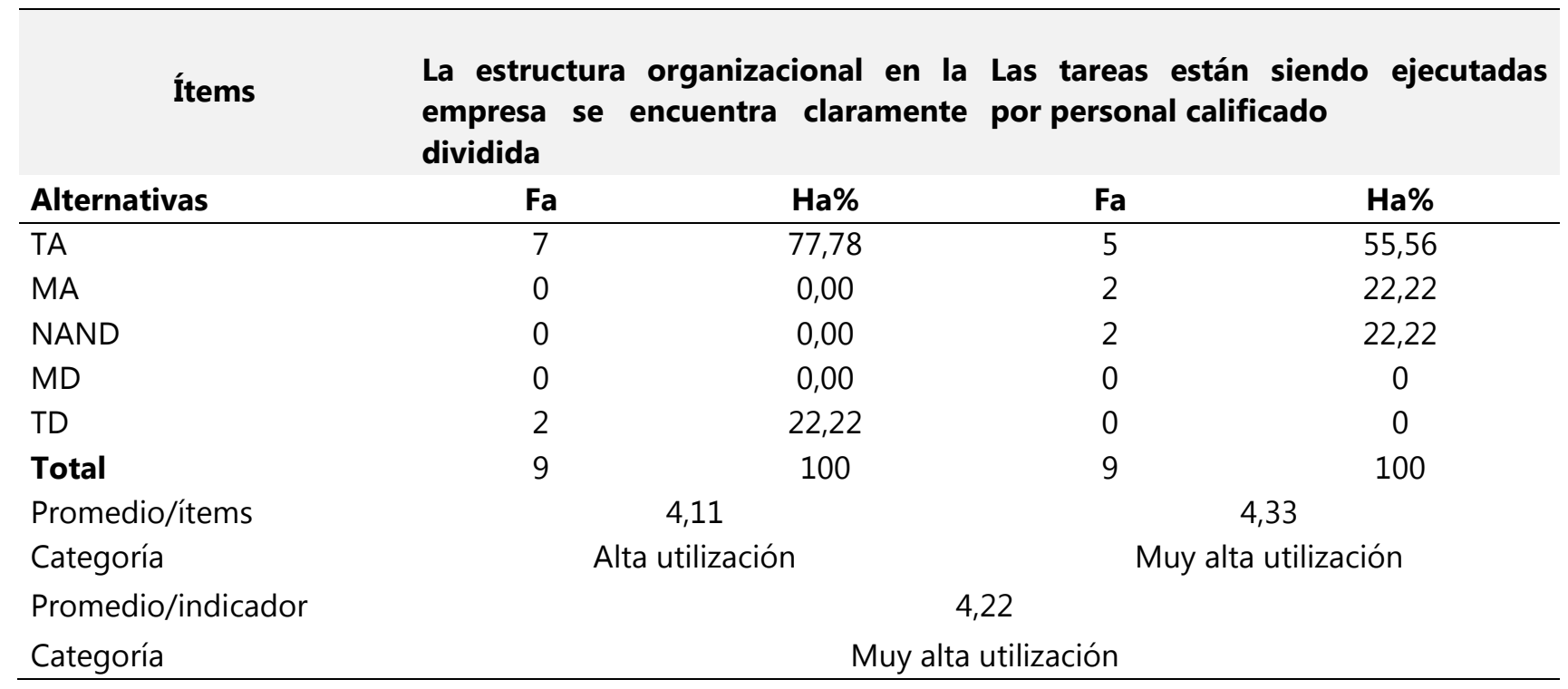

Fuente: Elaboración propia (2019)

Basado en los resultados, para las investigadoras, en las empresas analizadas la estructura organizacional es asumida como una disposición intencional de roles, en la que cada persona asume un papel que se espera que cumpla con el mayor rendimiento posible, su finalidad es establecer un sistema de papeles que han de desarrollar los gerentes de las empresas distribuidoras de productos lácteos, para trabajar juntos de forma óptima y que se alcancen las metas fijadas en la planificación.

Así las cosas, la estructura organizacional como proceso organizacional de la gestión logística permite la integración cliente proveedor a través de los departamentos, áreas y funciones que generan y transforman un bien o servicio, para ser entregado al cliente final de acuerdo a las exigencias del consumidor y estándares del mercado.

Seguidamente se hace referencia al indicador división del trabajo, ubicándolo en una categoría de muy alta utilización obteniendo un promedio de 4,33, como se muestra en la tabla 2, donde los encuestados están totalmente de acuerdo en un $44,44 \%$, al mismo tiempo el 33,33\% medianamente de acuerdo y solo un $22,22 \%$ no están de acuerdo ni en desacuerdo al considerar que el manual de cargos es tomado como prioridad para ejecutar las actividades.

También los resultados muestran con un $55,56 \%$ que los gerentes encuestados están totalmente de acuerdo, y de manera similar un $22,22 \%$ están medianamente y no están de acuerdo ni en desacuerdo en que las tareas se asignan a los equipos de trabajo, para que los mismos se turnen en las diversas tareas desempeñadas. 
Tabla 2. Indicador: División del trabajo

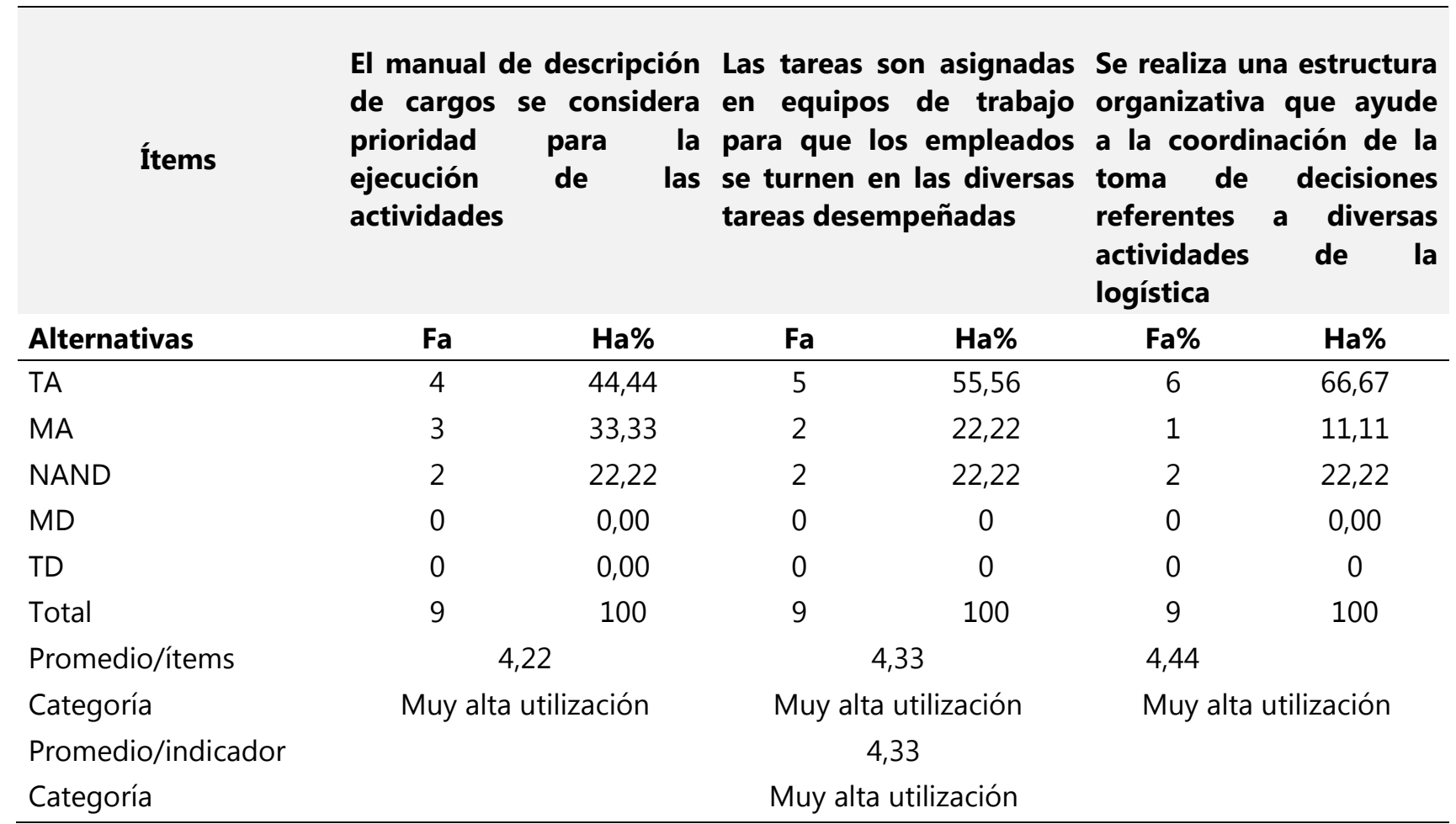

Fuente: Elaboración propia (2019)

Finalmente se observa en el indicador que el personal encuestado está totalmente de acuerdo con un $66,67 \%$, un $11,11 \%$ esta medianamente de acuerdo y solo un $22,22 \%$ no está de acuerdo ni en desacuerdo en manifestar que la empresa realiza una estructura organizativa que ayude a la coordinación de la toma de decisiones logísticas, que conlleve principalmente a la fragmentación de las actividades logísticas, tal como lo plantea Ballou (2004).

A juicio de las investigadoras, en las empresas analizadas existe una estructura organizativa que consiste en dividir $y$ fragmentar las actividades a equipos para que los empleados trabajen mediante el enfoque de equipos con el propósito de coordinar la toma de decisiones referentes a diversas actividades de la logística.
De ahí que, la división del trabajo como proceso organizacional de la gestión logística, permite el incremento de la productividad en determinadas tareas mediante la especialización en tareas concretas, siendo cada tarea realizada por una persona o grupo de personas por separado.

Para el indicador jerarquización, los resultados revelan un promedio de 4,28 ubicándose en la categoría de muy alta utilización, como se muestra en la Tabla 3, donde los encuestados están totalmente de acuerdo $77,78 \%$ y un $22,22 \%$ medianamente en desacuerdo en que los niveles jerárquicos están agrupados según el grado de autoridad.

Por otra parte, se visualizó que solo un $66,67 \%$ de los encuestados están totalmente de acuerdo, un $11,11 \%$ medianamente de acuerdo y solo un $22,22 \%$ no está en acuerdo ni en 
desacuerdo en considerar que la autoridad en la organización fluye desde el nivel ejecutivo hasta el nivel operativo.

Se observa claramente que en las empresas distribuidoras de productos lácteos estudiadas la jerarquización define la estructura de la empresa por medio de centros de autoridad y responsabilidad manteniendo un orden de rango, grado o importancia permitiendo que la relación fluya desde el más alto ejecutivo hasta el nivel más bajo, de acuerdo a lo planteado por Chiavenato (2007).

Según los resultados descritos, las investigadoras consideran que las empresas bajo estudio cuentan con un conjunto de órganos concentrados según el grado de autoridad y responsabilidad que posean, independientemente de la función que realicen.

En consecuencia, la jerarquización como proceso organizacional de la gestión logística, permite apalancarse en la especialización que suponen los proveedores externos, desarrollando un amplio espectro de outsourcing donde surgen importantes retos de coordinación y garantías, todo ello aderezado con el requerimiento del mantenimiento de los estándares de calidad, aspecto que ejercita un plano de certificaciones básico para el negocio.

Tabla 3. Indicador: Jerarquización

\begin{tabular}{|c|c|c|c|c|}
\hline Ítems & $\begin{array}{l}\text { Los nive } \\
\text { organizaci } \\
\text { de acuerd } \\
\text { que posea }\end{array}$ & $\begin{array}{l}\text { cos en la } \\
\text { lo agrupado } \\
\text { de autoridac }\end{array}$ & \multicolumn{2}{|c|}{$\begin{array}{l}\text { La autoridad fluye desde el más } \\
\text { alto ejecutivo hasta el nivel más } \\
\text { bajo }\end{array}$} \\
\hline Alternativas & $\mathrm{Fa}$ & $\mathrm{Ha} \%$ & $\mathbf{F a}$ & $\mathrm{Ha} \%$ \\
\hline TA & 7 & 77,78 & 6 & 66,67 \\
\hline MA & 0 & 0,00 & 1 & 11,11 \\
\hline NAND & 0 & 0,00 & 0 & 0,00 \\
\hline MD & 2 & 22,22 & 2 & 22,22 \\
\hline TD & 0 & 0,00 & 0 & 0 \\
\hline Total & 9 & 100 & 9 & 100 \\
\hline Promedio/ítems & \multicolumn{2}{|c|}{4,33} & \multicolumn{2}{|c|}{4,22} \\
\hline Categoría & \multicolumn{2}{|c|}{ Muy alta utilización } & \multicolumn{2}{|c|}{ Muy alta utilización } \\
\hline Promedio/indicador & \multicolumn{4}{|c|}{4,28} \\
\hline Categoría & \multicolumn{4}{|c|}{ Muy alta utilización } \\
\hline
\end{tabular}

Fuente: Elaboración propia (2019)

En lo referente al indicador opciones organizacionales los resultados lo ubicaron en una categoría de alta utilización con un promedio de 3,83 como se visualiza en la Tabla 4, e indica según las respuestas proporcionadas por los encuestados que solo un 22,22\% están totalmente de acuerdo, $11,11 \%$ medianamente de acuerdo, $22,22 \%$ no está de acuerdo ni en desacuerdo y $11,11 \%$ medianamente en desacuerdo en considerar que la organización se denomina de tipo informal en el momento que los responsables del cargo toman decisiones. 
Cabe acotar con un 88,89\% que los gerentes están totalmente de acuerdo y solo un $11,11 \%$ medianamente de acuerdo en considerar que la organización establece líneas claras de autoridad, lo que afirma que las empresas distribuidoras de productos lácteos analizadas clasifica su organización de tipo formal en donde cada gerente recibe y transmite todo lo que pasa en su área de competencia mediante canales de comunicación, conformando una estructura planificada con distintos niveles de jerarquía, autoridad y responsabilidad, tal y como sustenta Sierra (2013).

Tabla 4. Indicador: Opciones organizacionales

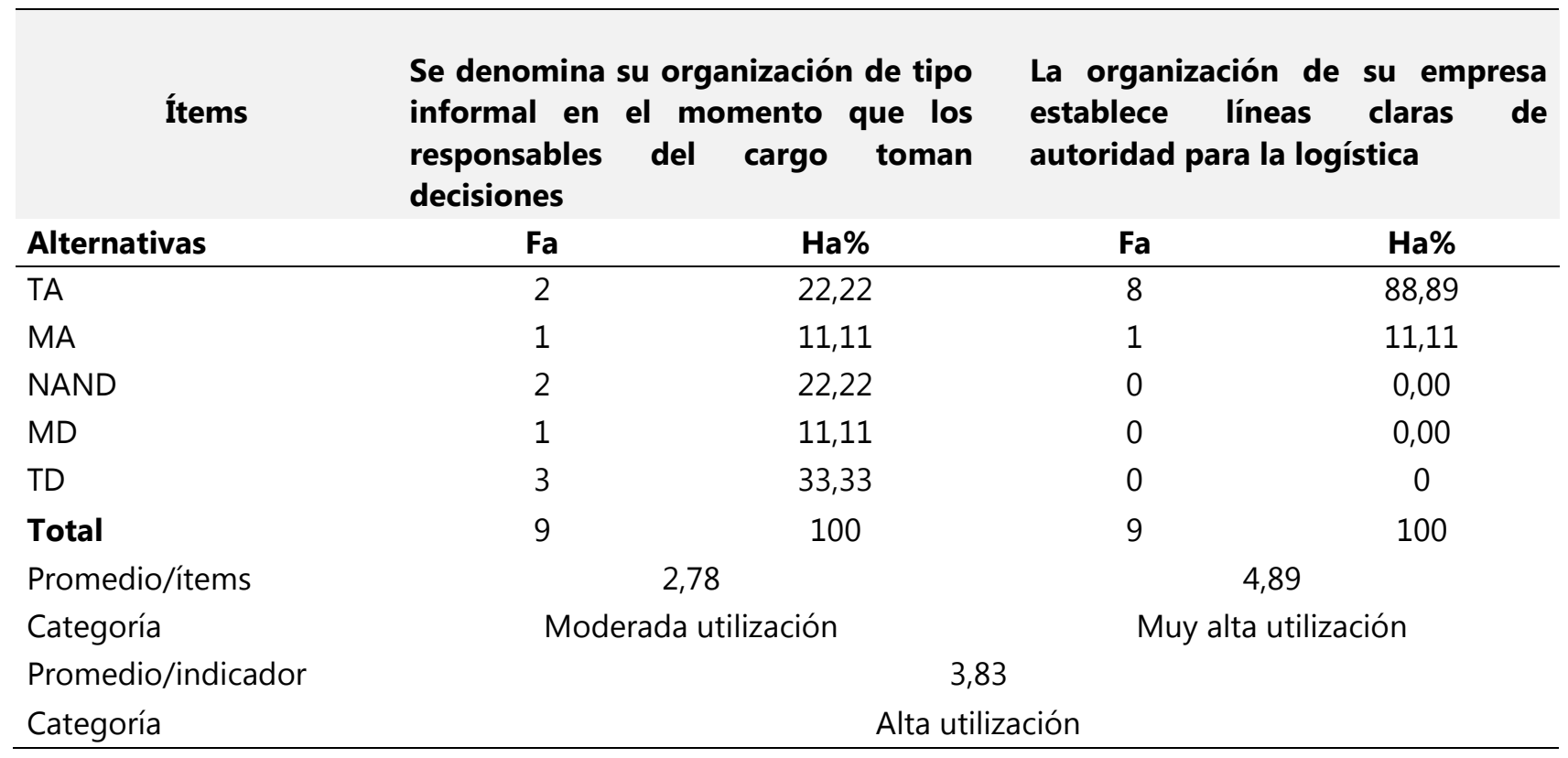

Fuente: Elaboración propia (2019)

También se puede observar, a juicio de las investigadoras, que el indicador opciones organizacionales no alcanza su nivel de excelencia, por lo que conlleva a estudiar exhaustivamente posibles mejoras en las empresas analizadas, con el fin de establecer líneas claras de autoridad y responsabilidad entre superiores y subordinados que establezca las relaciones entre los órganos, cargos y ocupantes con la finalidad de que sus objetivos sean alcanzados y su equilibrio interno sea mantenido, de acuerdo a lo manifestado por Chiavenato (2007).

Así pues, las opciones organizacionales como proceso organizacional de la gestión logística, es el modus operandi que les permite a las empresas distribuidoras de productos lácteos funcionar de acuerdo a su propia identidad y características empresariales.

Finalmente, la tabla 5, muestra el resumen para el análisis del proceso de organización logística, e indican según las respuestas proporcionadas por los gerentes encuestados un promedio de 4,17 ubicándose en una categoría de alta utilización. En términos generales, se desprende que existe un manejo óptimo en el proceso de organización de las empresas estudiadas a pesar de que el indicador opciones organizacionales refleja un promedio de 3,83 , lo que representa una posibilidad de mejora puesto que no llega al nivel de excelencia. 
Tabla 5. Dimensión: Proceso de organización

\begin{tabular}{lcc}
\hline DIMENSIÓN & PROMEDIO & CATEGORÍA \\
\hline Estructura Organizacional & 4,22 & Muy alta utilización \\
División del Trabajo & 4,33 & Muy alta utilización \\
Jerarquización & 4,28 & Muy alta utilización \\
Opciones Organizacionales & 3,83 & Alta utilización \\
TOTAL, PROMEDIO & $\mathbf{4 , 1 7}$ & ALTA UTILIZACIóN \\
\hline
\end{tabular}

Fuente: Elaboración propia (2019)

Estos resultados son coincidentes con lo postulado por Carranza y Sabria (2005), para quienes a través del proceso de la logística se planea e implanta, además controla de manera eficiente y económica el flujo y almacenamiento de materias primas, inventarios en proceso, productos terminados e información vinculada con ellos desde el punto de origen al punto de consumo con el propósito de adecuarse a los requerimientos del cliente.

Desde estas consideraciones, para las investigadoras en las empresas analizadas, el proceso de organización se enfoca en el esfuerzo de la logística, así pues, las empresas de productos lácteos analizadas, colocan las personas responsables para ejercer las actividades logísticas, de manera que se favorezca la coordinación entre ellas.

\section{CONCLUSIONES}

El proceso logístico cuenta con autonomía en el organigrama de las empresas modernas, encontrándose entre las áreas funcionales más determinantes. Su carácter multilateral hace que se requiera la integración de técnicas de trabajo diversas, es decir, equipos multidisciplinares, e interfuncionales. Es más, sus requerimientos de respuesta hacen necesaria una configuración plana, próxima a la posible fuente de incidencias.
Bajo estas premisas y según los resultados obtenidos, las empresas de productos lácteos ubicadas en el municipio Cabimas, manejan una alta utilización el proceso organizacional, puesto que el sistema se caracteriza por establecer una estructura organizacional adecuada, coordinar la división del trabajo a través de la fragmentación de las actividades, y mantener una clara definición de la estructura de la empresa a través de la jerarquización y las opciones organizacionales.

Así que, estos estudios tienen un impacto positivo en las empresas distribuidoras de productos lácteos, no sólo porque permite ahorros significativos en los procesos logísticos sino también porque permite estandarizar procesos y crear historia en las decisiones que en una gran mayoría de los casos son tomadas empíricamente.

\section{REFERENCIAS}

Ávila R. (2010). Estudio de factibilidad para la implementación de un proceso logístico integral en un negocio de alimentos refrigerados en el área de Guatire. Disponible en: https://pdfslide.net/documents/estudio-defactibilidad-para-la-implementacion-de-unproceso-.html 
Ballou, R. (2004). Logística. Administración de la cadena de suministro. 5ta Edición. Naucalpan de Juárez, México

Carranza, O. y Sabria, F. (2005). Logística: Mejores Prácticas en Latinoamérica. 2da edición. International Thomson Editores. Distrito Federal, México

Castellanos, A. (2009). Manual de la gestión logística del transporte y distribución de mercancías. Ediciones Uninorte. Colombia
Chiavenato, I. (2007). Administración en los nuevos tiempos. Editorial limusa, S.A. de C.V. México

Gavilanes, J. (2012). Niveles Jerárquicos de la Empresa u Organización Disponible en: http://nivelesdelaempresa.blogspot.com/

Sierra, I. (2013). Administración, clasificación de las organizaciones. Disponible en: https://es.slideshare.net/loreeuniica/admini stracin-25050507 